\title{
Revistas Depredadoras: Un Mal Vertiginoso con Ramificaciones Intelectuales
}

\author{
Depredatory Journals: An Aberrant Phenomenon with Intellectual Ramifications
}

\author{
Alfonso Martínez-Taboas ${ }^{1^{*}}$ \\ 1 Universidad Interamericana de Puerto Rico, Puerto Rico. (D) https://orcid.org/0000-0001-8331-0423 \\ * Correspondencia: alfonsomtaboas@gmail.com
}

Recibido: 28 diciembre 2021 | Aceptado: 19 enero 2022 | Publicado: 23 enero 2022

WWW.REVISTACARIBENADEPSICOLOGIA.COM

\section{Citar como:}

Martínez-Taboas, A. (2022). Revistas depredadoras: Un mal vertiginoso con ramificaciones intelectuales. Revista Caribeña de Psicología, 6, e6165. https://doi.org/10.37226/rcp.v6i1.6165

\section{RESUMEN}

En este artículo discuto con amplitud el fenómeno de las revistas depredadoras, las cuales no poseen las características que tienen revistas académicas y profesionales. Así, por ejemplo, la revisión de pares no existe o es fraudulenta, no están asociadas a organizaciones de reputación reconocida y los editores y miembros de la Junta Editora son desconocidos o son identidades inventadas. El propósito es aceptar el artículo, a veces en cuestión de horas o días, para recolectar dinero por la publicación. Este tipo de revista ha crecido exponencialmente en los últimos años, abarcando también la psiquiatría, la psicología y las neurociencias. En este artículo se presentan características comunes de las revistas depredadoras y estudios realizados sobre cómo se lleva a cabo este proceso fraudulento. Se resalta que este tipo de publicación ilegítima puede corromper el conocimiento científico y atentar contra las bases epistemológicas de las ciencias naturales y sociales.

Palabras Claves: fraude, publicaciones científicas, revistas depredadoras

\section{ABSTRACT}

In this article, I discuss the phenomenon of depredatory journals, which do not possess the essential characteristics of academic and professional journals. For example, these journals do not use peer review, or the process of reviewing an article is entirely fraudulent, they are not associated with well-known and respected professional associations, and the editors and members of the editorial board are unknown people or invented. The main purpose of depredatory journals is to accept any submitted article to collect money in a matter of a few days. This type of journal has increased exponentially in the last decade, affecting psychiatry, neurosciences, and psychology. In this article, I present some of the more constant characteristics of these journals and empirical studies that illustrate their modus operandi. I highlight that this type of journal can be devastating to the scientific community potentially corrupt legitimate scientific knowledge.

Keywords: depredatory journals, fraud, scientific publications 


\section{Introducción}

Diversos epistemólogos y filósofos de la ciencia han planteado que la actividad científica tiene como cimiento un grupo de personas, altamente cualificadas, que exponen sus ideas y datos a una colectividad, a su vez, poseen conocimiento especializado para analizar, criticar o corroborar lo que se plantea (Lack \& Rousseau, 2016). Tal y como lo ha expuesto magistralmente Longino (1990, 2001), la objetividad de la búsqueda científica se mantiene al entenderla como un proceso social en vez de uno individual. Por ejemplo, proyectos como el Genoma Humano implican la colaboración sistemática de un grupo enorme de científicos trabajando en conjunto en un proyecto en particular (McCain, 2016).

La actividad científica es distinta a lo que sucede en otros saberes. En las ciencias, sean naturales o de observación, las metodologías científicas implican que los supuestos puedan ser corroborados empíricamente, que los planteamientos teóricos permitan su posible refutación y que los hallazgos sean planteados a la comunidad científica para que puedan ser replicados. Sin embargo, hay otros saberes que parten de premisas extra-científicas. Por ejemplo, en la teología, los supuestos muchas veces no son posibles de corroborar empíricamente. Muchos postulados teológicos deben aceptarse por fe y por dogmas arraigados en ese saber. Ejemplos serían: el concepto de la trinidad, la inmaculada concepción, y el purgatorio dentro del catolicismo.

La ciencia, sin embargo, es una creación humana que tiene como meta analizar, estudiar y escudriñar diversos objetos de estudios, que pueden ir desde la naturaleza que nos rodea, hasta los patrones de las sociedades humanas y sus habitantes. Por lo tanto, la ciencia es eminentemente una actividad humana y como tal está a expensas de sus fragilidades: errores, fraude, inconsistencias y equivocaciones (Boetto et al., 2021).

Un aspecto importante y que distingue la actividad científica de otros saberes es la necesidad imperiosa de someter las ideas y datos a la comunidad internacional de científicos en cierta área. El propósito de esta exposición es cerciorarse de que lo que se postula pueda ser replicado por otros investigadores, preferiblemente por colegas internacionales. Resulta imperativo en la ciencia entender cuáles variables específicas son causales o responsables de ese resultado.

En psicología son incontables los ejemplos que se pueden brindar. En los años 50 dominaban los modelos psicoanalíticos (Paris, 2005). En esa misma década surgió el modelo de desensibilización sistemática para tratar trastornos fóbicos (Wolpe, 1954, 1958) que fue rechazado y criticado por los principales psicoanalistas. Para los psicoanalistas, atacar el síntoma directamente traería sustituciones de síntomas. El debate sobre la eficacia y efectividad de este modelo hizo que, para las décadas de los 60 y 70 aumentaran los estudios sistemáticos en pacientes con fobias severas, principalmente en Londres (Gelder et al., 1967; Marks, 1981). Estos estudios, fueron una muestra de cómo la ciencia no es estática, sino que está en constante movimiento y evolución; así, pues, de la desensibilización sistemática se derivaron las terapias de exposición prolongada, las cuales al día de hoy son terapias de primera línea para las fobias y trastornos de ansiedad.

Lo crucial para la gestación de este análisis es activar las mentes críticas y especializadas de las personas que colectivamente componen la ciencia. Una de las maneras más efectivas de lograr esto es presentar las ideas en formato escrito en revistas científicas arbitradas. ¿Qué significa esto? Significa que los científicos tienen que presentar sus teorías o datos a la comunidad científica en revistas especializadas en donde varios expertos de dicho campo leerán o revisarán el trabajo sometido con una mirada crítica y sistemática. La meta es examinar el manuscrito para anotar y señalar posibles errores, deficiencias o inconsistencias. Típicamente las revistas científicas logran este cometido utilizando lo que se conoce como una revisión a ciegas (blind review), donde los revisores desconocen la identidad del autor (es) y viceversa. De esta manera se minimiza la posibilidad de brindar revisiones sesgadas. Este proceso de revisión de pares típicamente puede tomar entre dos a seis meses, ya que lo usual es que cada artículo sea revisado por dos a cuatro expertos en el tema. Por lo tanto, la revisión de pares puede ser definida como "el proceso de someter el trabajo escrito de un autor, al escrutinio de otras personas que son expertos en ese tema" (Kelly 
et al., 2014). Precisamente esta es la característica esencial de las publicaciones científicas.

Debido a esta metodología, la mayoría de los artículos enviados a revistas técnicas y de buena reputación científica, no cumplen con el estándar establecido y son rechazados. Khadilkar (2018) ha señalado que del $80-85 \%$ de los artículos enviados a revistas científicas son rechazados. En algunas revistas de alta reputación, puede llegar al 95\%. Cuando un artículo es enviado a una revista científica menos competitiva, la tasa de rechazo entonces suele estar en el 50\% (Kelly et al., 2014).

\section{Revistas Depredadoras: ¿Qué Son?}

Las revistas depredadoras son entes de publicación de acceso abierto que no siguen el formato legítimo de publicar trabajos con metodologías aceptables o rigurosas en el campo de las ciencias. Por lo tanto, lo que publican son artículos de pobre o escasa calidad que se desvían marcadamente de las mejores prácticas editoriales. Lo usual es que cursan invitaciones a innumerables personas que han publicado anteriormente a que sometan sus artículos a dichas revistas. Si el artículo es enviado, no pasa por el proceso riguroso de revisión de pares o hacen un simulacro fraudulento del mismo. El artículo típicamente es publicado en días o pocas semanas luego de enviarlo al editor. Luego de ser aceptado, se le pide al autor que pague una tarifa para su publicación. Desde luego, la meta principal de las revistas depredadoras es obtener ganancias económicas de este proceso. El producto final publicado tiene un potencial enorme de ser deficiente pues no ha sido revisado de manera crítica y detallada por expertos en el tema. Por lo tanto, en las revistas depredadoras la tasa de rechazo es prácticamente cero.

Una de las primeras personas que dio la voz de alerta sobre las revistas depredadoras fue un bibliotecario llamado Jeffrey Beall (2014) de la Universidad de Colorado. Posterior a esto, continuaron surgiendo estimados de revistas depredadoras, calculándose en unas 1,100 (Jiménez-Contrera \& Jiménez-Segura, 2016).

Las revistas depredadoras se han aprovechado de un ambiente intelectual donde muchos académicos y científicos tienen que publicar cierto número de artículos anualmente para cumplir compromisos con su contrato laboral. Este fenómeno se agudiza en académicos jóvenes y con poca experiencia sometiendo artículos a revistas científicas. De hecho, en el análisis llevado a cabo por Xia et al. (2015) se encontró que los que tienden a enviar artículos a revistas depredadoras son académicos jóvenes, con poca experiencia en su trabajo y de países en desarrollo. Las revistas depredadoras son una aparente solución a esta situación, pues es casi seguro que cualquier tipo de trabajo, aun esos con deficiencias obvias, serán publicados. Para añadir complejidad al asunto, muchas de estas revistas cuentan con nombres sofisticados tales como: Journal of Information System Management, International Review of Social Psychology, Systems Science Journal, entre muchas otras.

Como mencioné, todas las revistas depredadoras se publican con el formato de acceso abierto (open access) en donde el contenido del artículo podrá ser leído por cualquier individuo en el planeta. No hay duda que el formato de acceso abierto es una evolución positiva en la comunicación científica. De hecho, debe aclararse que hay revistas científicas de alta reputación que utilizan este formato; una de las más reconocidas es Plos One. Por lo tanto, las revistas depredadoras son parasitarias de un sistema que ya existía y que ha sido exitoso.

\section{¿Cómo las Podemos Reconocer?}

Siguiendo autores como Bartholomew (2014), Cobey et al. (2018) y Looi y Owens-Walton (2020), se puede identificar que las revistas depredadoras tienen muchas de las siguientes características:

1. Son en formato de acceso abierto (open access).

2. Le cobran al autor por la publicación, en muchas ocasiones con tarifas relativamente bajas (\$150.00 - \$400.00). En revistas profesionales con un factor de impacto alto, la publicación puede sobrepasar los $\$ 1,000.00$. De hecho, es bastante común que el autor no esté enterado de que tendrá que pagar para ver su artículo publicado, ya que se le informa posterior a su aceptación.

3. La revisión y aceptación del artículo se realiza en días o una semana.

4. El resultado de la revisión es que el artículo no necesita cambios o se sugieren cambios mínimos. 
5. Los editores son personas desconocidas o ficticias en el campo.

6. La Junta Editora está compuesta de personas desconocidas o ficticias en el campo.

7. La revisión la tiende a realizar el editor. No hay evidencia de la intervención de revisores externos.

8. No hay ninguna organización profesional reconocida afiliada a la revista.

9. Pocas veces aparecen en listas de indexación como SCOPUS y Medline. En el caso de la psicología sería PsycINFO.

10. Le envían directamente al autor invitaciones para someter artículos o para ser editor de una revista. En ocasiones los autores reciben múltiples invitaciones para publicar en dicha revista. Esta es una práctica poco común en revistas científicas (Moher \& Srivastava, 2015), ya que lo usual es realizar lo que se conoce como un "call for papers" (invitación para someter) la cual se publica en las propias páginas de la revista profesional.

11. No utilizan medidas de factores de impacto, excepto algunas que utilizan el Index Copernicus Value, el cual ha sido extensamente criticado. También puede suceder que citan datos fabricados de factor de impacto.

12. Muchos miembros de las Juntas Editoras no pueden ser localizados o simplemente no existen.

13. Le piden al autor que someta el artículo a través de una dirección por email, en vez de un sistema estandarizado de someter artículos.

14. Menciona o promete que la revisión se hará rápido.

15. El email de la casa publicadora es genérico (por ejemplo, un Gmail, Hotmail o Yahoo).

16. No hay una política sobre artículos que pueden pasar por retracciones, plagio, errata y correcciones. Recordemos que retracciones es cuando un artículo es publicado y posteriormente se encuentran errores notables o datos falsificados que obligan a retirar el artículo de las páginas de la revista.

17. Ausencia de estándares usuales (no hay DOI o ISSN, y si los tienen, son falsos).

18. Sus servidores suelen estar mayormente localizados en Nigeria, India, China, Irán y Pakistán.
La práctica de lanzar invitaciones es una muy común en las revistas depredadoras. Usualmente obtienen los nombres y direcciones electrónicas de artículos que han sido publicados previamente en revistas arbitradas. El racional de las invitaciones en sí es dudoso pues la mayoría de las revistas con buena reputación solo publican un número reducido de los artículos que reciben, por lo que no tiene sentido invitar autores cuando ya no tienen espacio para publicar.

Muchas veces la invitación de un editor de una revista depredadora dice algo como lo siguiente: "Encontré un artículo suyo titulado El Trastorno Bipolar y su Relación con Intentos Suicidas y como editor entiendo que usted sería un excelente candidato para someter a nuestra revista".

\section{Mi Experiencia}

Debido a que soy un autor que publica artículos profesionales prolíferamente, he recibido docenas de invitaciones por parte de revistas depredadoras para someter artículos, ser miembro de una Junta Editora o ser editor. Por ejemplo, recientemente recibí lo siguiente: "Saludos. La Open Journal of Geriatrics está aceptando artículos para el próximo volumen (Vol. 4). Le pedimos a usted su apoyo y contribución, sometiendo su manuscrito y lo publicaremos a un bajo costo ( $50 \%$ descuento). Si tiene alguna duda se comunica con nosotros. Aprecios, XXXXX, Gerente de la revista". Al hacer una búsqueda de esta revista, la misma es publicada por Sryahwa Publications, la cual aparece en listados de revistas depredadoras. No solo esto, nótese que el que envía la invitación no es un académico, sino un supuesto gerente de una compañía.

\section{El Escenario en las Revistas Psicológicas y Psiquiá- tricas}

La American Psychological Association (APA, 2014) ha informado que el $41 \%$ de sus revistas tiene una tasa de rechazo mayor al $70 \%$. En promedio la tasa es mayor a 50\%. La revista con menos tasa de rechazo es Group Dynamics con un 39\% y la mayor es Counseling Psychology con $98 \%$.

Beall y DuBois (2016) recientemente lanzaron una advertencia a los psicólogos en los EE. UU. Enfatizaron en que las casas de publicación de revistas depredadoras están haciendo acercamientos insistentes a innumerables psicólogos y científicos sociales para 
que publiquen en dichas revistas. Estos autores indican que las casas publicadoras de revistas depredadoras han engañado a innumerables autores, haciéndoles creer que están publicando en revistas de reputación en el campo. Añaden Beall y DeBois: “Estas casas usan técnicas avanzadas de spam, alcanzando así a muchos investigadores a través de una invitación personal que alaba lo que el investigador ha publicado e invitándolo a someter sus artículos" (p. 42).

El impacto negativo de publicar en revistas depredadoras es devastador. Se estarían publicando estudios deficientes que pueden ser citados como un conocimiento legítimo cuando no lo es. De hecho, dichos artículos tienen un potencial enorme de corromper el conocimiento en ciertas áreas. Sobre este punto, recientemente Omer et al. (2019) realizaron una búsqueda de revistas identificadas como depredadoras que se dedican al campo de la psiquiatría. La búsqueda arrojó 126 revistas depredadoras, lo que es un número alarmante. Del 2007 al 2019 esas revistas publicaron 6,925 artículos. En total, publicaron en dichas revistas 26,363 investigadores, provenientes de 133 países del mundo. Por su parte, Manca et al., (2017) realizaron un estudio sobre el número de revistas y artículos publicados en revistas depredadoras sobre neurología y neurociencias. Resulta alarmante que identificaron 101 revistas depredadoras en neurología y 87 revistas en neurociencias.

\section{¿Qué se Recomienda?}

En primer lugar, si vamos a someter un artículo debemos conocer la trayectoria científica o académica de dicha revista. Mayormente dichas revistas tienen menos de una década de comenzar a publicarse. También podemos buscar información de la reputación de los editores y los asistentes del editor, y buscar si investigadores de renombre tienden a publicar en sus páginas. En caso de dudas, se debe consultar a un colega experto en dicha área.

En segundo lugar, no debemos aceptar invitaciones de dichas casas editoras para servir de editores o pertenecer en sus Juntas Editoriales. Al hacerlo, estamos respaldando intentos de desvirtuar nuestra ciencia psicológica. En tercer lugar, debemos educar a nuestros estudiantes e investigadores emergentes sobre esta situación y discutirlo en clases de investigación. Esto puede dar base a debates interesantes sobre la naturaleza de la ciencia y su distinción de las pseudociencias (Gordin, 2021). En Puerto Rico, el tema de las revistas depredadoras en psicología y psiquiatría no ha sido discutido en las revistas académicas ni tampoco se ha abierto un foro en las convenciones de la Asociación de Psicología de Puerto Rico. Esto es preocupante, pues estas revistas ya se han infiltrado en estos campos.

En cuarto lugar, cuando un investigador busca promoción en una institución universitaria, se deben escudriñar sus publicaciones y de dónde provienen. Ya se sabe que personas que buscan permanencia académica podrían publicar en dichas revistas y así cumplir con las expectativas de la institución de publicar cierto número de artículos por año. Sin embargo, esta situación puede ir cambiando ya que comienza a haber precedentes donde la facultad que somete artículos en revistas depredadoras puede ser despedidos de su puesto (Simón, 2016). No solo dicha publicación puede dar base a un despido, sino también al descrédito de su reputación e imagen profesional. Por ejemplo, recientemente, Pond et al. (2019) escudriñaron los curriculum vitae de nueve personas que estaban aplicando a una plaza de facultad en la Universidad del Estado de Tennessee. Para su sorpresa, el 17.6\% de los artículos de estos candidatos estaban publicados en revistas depredadoras. No solo eso, sino que tres de estos candidatos a facultad tenían más del $80 \%$ de sus artículos publicados en dichas revistas.

Más allá de esfuerzos personales, las instituciones académicas deben crear espacios en los cuales se discuta con amplitud y rigurosidad el fenómeno de las revistas depredadoras en los currículos de ética y metodología. Además, podrían crear algún taller sobre este tema para concientizar y sensibilizar a la facultad y estudiantes de los peligros de publicar en estas revistas.

\section{Revistas Depredadoras Puestas a Prueba}

Varios investigadores han realizado la tarea de someter a revistas depredadoras trabajos que son creados con múltiples deficiencias. Simón (2016), por ejemplo, sometió a la revista British Arts and Social Science un manuscrito que contenía errores marcados: por ejemplo, no sometió las referencias con un orden lógico. En cuestión de 3 días el editor le escribió aceptando el artículo sin correcciones y felicitándolo por un artículo que deliberadamente había sido escrito con múltiples deficiencias. Más adelante, Simón 
decide someter otro manuscrito a dicha revista que consistió de plagiar innumerables textos de Freud, Steve Jobs y autores de psicología evolutiva, sin prácticamente ningún racional teórico ni empírico. Unos pocos días más tarde el artículo fue aceptado sin cambios.

Por otro lado, Hakami (2017) publicó en detalle cómo fue el proceso de someter un artículo ridículo y repleto de errores a una revista depredadora. La misma lleva el título de International Journal of Social Science and Humanities Research. El artículo fue sometido con "errores mayúsculos" en términos estadísticos, múltiples errores gramaticales, tablas con errores obvios y se indicó que la investigación no había pasado por ningún comité de ética. Por ejemplo, en el resumen del artículo se indicó que participaron 800 personas. Pero en la sección de método se decía que eran 500. Asimismo, cuando se decía que había diferencias estadísticamente significativas, no se indicaba el valor de las diferencias y las escalas que se usaron tenían un Cronbach de alfa ridículamente inaceptable (0.12). Al día siguiente de someter el artículo, Hakami recibe una carta informándole que su artículo había sido aceptado sin cambios. También se le informó que depositara \$50.00 a una cuenta bancaria.

Asimismo, dos científicos cognitivos escribieron un artículo en donde se repetía cientos de veces la oración "Get me off your fucking mailing list" (Sáquenme de su maldita lista de correos). Incluyeron dos gráficas en donde el contenido repetía dicha oración como la variable principal. El artículo fue enviado a la revista International Journal of Advanced Computer Technology. El artículo fue aceptado como "excelente" por el editor. Se les pidió de inmediato a los autores que pagaran $\$ 150.00$ (Mazieres \& Kohler, 2014).

Por su parte, Stromberg (2014) detalla el estudio realizado por Tom Spears y que fue sometido a varias revistas depredadoras. Ocho de éstas aceptaron el artículo sin ningún señalamiento. El artículo utilizó un plagio masivo y el compendio fue un manuscrito sin lógica ninguna. El tema era la relación entre el planeta Marte, el cáncer y los terrenos.

Un último ejemplo, algo distinto, es el publicado por Sorokowski et al. (2017). Estos investigadores provienen del Departamento de Psicología de la Universidad de Polonia. Se envió a 360 revistas, algunas legítimas y otras depredadoras, el perfil de una doctora llamada Anna O. Szust (en polaco Oszust quiere decir fraude) que deseaba ser editora de una revista científica. El perfil creado era totalmente inaceptable para ser editora. Por ejemplo, nadie había citado su trabajo, su curriculum vitae no incluía artículos en revistas académicas, no tenía ninguna experiencia como revisora de artículos, y los capítulos que sometió simplemente no existían en ninguna base de datos. De hecho, las casas publicadoras eran inventadas. A pesar de todas estas deficiencias, 40 de las revistas depredadoras nombraron a Anna O. Szust como Editora en Jefe en cuestión de horas o días. Esto no sucedió en ninguna de las revistas de reputación académica.

Simón (2016) realizó un análisis más detallado del modus operandi de estas revistas. Sometió un manuscrito a una revista depredadora en el área de la psicología titulada Open Psychology Journal. La misma es publicada en China por una casa publicadora llamada Bentham Open. Al examinar su contenido encontró que cada volumen consistía de solo dos o tres artículos y con innumerables errores gramaticales. Otro ejemplo patente de fraude sucedió con la revista depredadora titulada Psychology (Simon, 2016). En dicha revista se publicaron artículos ya publicados en una revista publicada por APA titulada Journal of Personality and Social Psychology. Posteriormente los artículos fueron retirados. Esta revista también es publicada en China.

Revelador también es el estudio de Bohannon (2013) quien envió a 304 revistas de acceso abierto un artículo con un nombre falso (Ocorrafoo Cobange), con una institución falsa (Wassee Institute of Medicine) y con un contenido científico que "contenía tantos errores graves que un revisor competente hubiera identificado el artículo como uno totalmente deficiente e imposible de ser publicado" (p.61). En total, 157 de estas revistas aceptaron el artículo.

\section{Conclusión}

La ciencia es una actividad social y colectiva que ha sido exitosa en parte por producir avances significativos en diversas áreas tales como la medicina, las ciencias sociales y la tecnología. Pero estos avances se han logrado mediante un monitoreo intenso y crítico a través de las publicaciones científicas. Las revistas depredadoras tratan de evadir este escrutinio. Al no utilizar de manera rigurosa la revisión de pares y 
darle énfasis a aumentar su capital económico, se está entonces desvirtuando el propósito de lo que es una publicación válida y confiable. Esto ya de por sí es sumamente peligroso ya que estudios deficientes, fraudulentos y erráticos pueden pasar como conocimiento confiable, cuando no son otra cosa que pseudociencia.

El propósito de este artículo ha sido alertar a colegas latinoamericanos y caribeños de la existencia creciente de estas revistas. La tarea no es sencilla pues muchos investigadores o autores no conocen el universo de revistas legítimas y arbitradas en su campo. Por lo tanto, debemos todos combatir y denunciar estas prácticas ilegítimas de actividad intelectual. La credibilidad y reputación de lo que enseñamos y creemos no puede basarse en artículos y escritos potencialmente fallidos.

Financiamiento: La presente investigación no fue financiada por alguna entidad ni patrocinador.

Conflicto de Intereses: No existen conflictos de intereses de parte de los autores de la investigación.

Proceso de Revisión: Este estudio ha sido revisado por pares externos en modalidad de doble ciego.

\section{REFERENCIAS}

Abramowitz, J., Deacon, B., \& Whiteside, S. (2019). Exposure therapy for anxiety. Guilford.

American Psychological Association. (2014). Summary report of division journals operations. American Psychologist, 69, 531-532

Bartholomew, R. E. (2014). Science for sale: The rise of depredatory journals. Journal of the Royal Society of Medicine, 107, 384385. https://doi.org/10.1177/0141076814548526

Beall, J. (2014). Unintended consequences: The rise of predatory publishers and the future of scholarly publishing. Editorial Office News, 2014, 4-6.

Beall, J., \& DuBois, J. M. (2016). Scholars beware. Monitor on Psychology, 47(4), 42.

Boetto, E., Golinelli, D., Carullo, G. \& Fantini, M. (2021). Frauds in scientific research and how possibly to overcome them. Journal of Medical Ethics, 47, e19. https://doi.org/10.1136/medethics-2020-106639

Bohannon, J. (2013). Who's afraid of peer review? Science. 342(6154), 60-65. http://doi.org/10.1126/science.342.6154.60

Cobey, K., Lalu, M., Skidmore, B., Ahmadzai, N., Grudniewicz, A., \& Moher, D. (2018). What is a predatory journal? F1000 Research, 7, 1000. https://doi.org/10.12688/f1000research.15256.2
Gelder, M. G., Marks, I. M., \& Wolff, H. (1967). Desensitization and psychotherapy in the treatment of phobic states: A controlled inquiry. British Journal of Psychiatry, 113, 53-73. https://doi.org/10.1192/bjp.113.494.53

Gordin, M. D. (2021). On the fringe: Where science meets pseudoscience. Oxford University Press.

Hakami, R. (2017). Predatory journals: Write, submit, and publish the next date. Skeptical Inquirer, 41 (5), 25-29.

https://skepticalinquirer.org/2017/09/predatory-journalswrite-submit-and-publish-the-next-day/

Jiménez-Contreras, E., \& Jiménez-Segura, J. J. (2016). Las revistas depredadoras, una nueva epidemia científica. Ciencia y Enfermería, 22(2), 7-12. https://revistas.udec.cl/index.php/cienciayenfermeria/article/view/168

Kelly, J., Sadeguieh, T., \& Adeli, K. (2014). Peer review in scientific publications: Benefits, critiques, \& a survival guide. The Journal of the International Federation of Clinical Chemistry and Laboratory Medicine, 25(3), 227-243.

https://www.ncbi.nlm.nih.gov/pmc/articles/PMC4975196/pdf/ejifcc-25-227.pdf

Khadilkar, S. (2018). Rejection blues: Why do research papers get rejected? The Journal of Obstetrics and Gynecology of India, 68, 239-241. https://doi.org/10.1007/s13224-018-1153-1

Lack, C. W., \& Rousseau, J. (2016). Critical thinking, science, and pseudoscience. Springer.

Longino, H. (1990). Science as social knowledge: Values and objectivity in scientific inquiry. Princeton University Press.

Longino, H. (2001). The fate of knowledge. Princeton University Press.

Looi, C., \& Owens-Walton, O. (2020). Sharks: Predatory journals and conferences in psychiatry, neurology and neuroscience. Australian and New Zealand Journal of Psychiatry, 29, 1-2. https://doi.org/10.1177/0004867420919154

Manca, A., Martinez, G., \& Cugusi, L. (2017). The surge of predatory open-access in neurosciences and neurology. Neuroscience, 353, 166-173. https://doi.org/10.1016/j.neuroscience.2017.04.014

Marks, I. M. (1981). The cure and care of neurosis. Wiley Publications.

McCain, K. (2016). The nature of scientific knowledge. Springer.

Mazieres, D., Kohler, E. (2014). Get me off your fucking mailing list. Stanford Secure Computer Systems Group, Stanford University. Retrieved November 22, 2014. https://www.scs.stanford.edu/ dm/home/papers/remove.pdf

Moher, D., \& Srivastava, A. (2015). You are invited to submit... BMC Medicine, 13, 180. https://bmcmedicine.biomedcentral.com/articles/10.1186/s12916-015-0423-3\#citeas

Omer, J., Mohammed, S.H., \& Salih, R.Q. (2019). Predatory journals in psychiatry. The Lancet. Psychiatry, 6, 564-565. https://openexcellence.org/predatory-journals-in-psychiatry/

Paris, J. (2005). The fall of an icon: Psychoanalysis and academic psychiatry. University of Toronto Press. 
Pond, B., Brown, S., Stewart, D., Roane, D., \& Harirforoosh, S. (2019). Faculty applicants' attempt to inflate CVs using predatory journals. American Journal of Pharmaceutical Education, 83 (1), 12-14. https://doi.org/10.5688/ajpe7210

Simón, A., (2016). Pitfalls of predatory journals: A personal account. Comprehensive Psychology, 5, 1-5. https://doi.org/10.1177\%2F2165222816631691

Sorokowski, P., Kulczycki, E., Sorokowska, A., \& Pisanski, K. (2017). Predatory journals recruit fake editor. Nature, 543, 481483. https://doi.org/10.1038/543481a

Stromberg, J., (2014). A reporter published a fake study to expose how terrible some scientific journals are. Vox, April 24. https://www.vox.com/2014/4/24/5647106/a-reporter-published-a-fake-study-to-expose-how-terrible-some

Wolpe, Joseph. (1954). Reciprocal inhibition as the main basis of psychotherapeutic effects. Archives of Neurology and Psychiatry, 72, 205-226. https://doi.org/10.1001/archneurpsyc.1954.02330020073007

Wolpe, J. (1958). Psychotherapy by reciprocal inhibition. Stanford University Press.

Xia, J., Harmon, J., Connolly, K., Donnelly, R., Anderson, M., Howard, D. (2015). Who "publishes" in predatory journals? Journal of the Association for Information Science and Technology, 66, 1406-1417. https://doi.org/10.1002/asi.23265

Obra bajo licencia de Creative Commons Atribución 4.0 Internacional (CC BY 4.0). (C) 2022 Autores. 